\title{
Univalent harmonic mappings
}

\author{
by Albert E. Livingston (Newark, Del.)
}

\begin{abstract}
Let $a<0, \Omega=\mathbb{C}-(-\infty, a]$ and $U=\{z:|z|<1\}$. We consider the class $S_{H}(U, \Omega)$ of functions $f$ which are univalent, harmonic and sense preserving with $f(U)=\Omega$ and satisfy $f(0)=0, f_{z}(0)>0$ and $f_{\bar{z}}(0)=0$. We describe the closure $\overline{S_{H}(U, \Omega)}$ of $S_{H}(U, \Omega)$ and determine the extreme points of $\overline{S_{H}(U, \Omega)}$.
\end{abstract}

1. Introduction. Let $S_{H}$ be the class of functions $f$ which are univalent sense preserving harmonic mappings of the unit disk $U=\{z:|z|<1\}$ and satisfy $f(0)=0$ and $f_{z}(0)>0$. Let $F$ and $G$ be analytic in $U$ with $F(0)=G(0)=0$ and $\operatorname{Re} f(z)=\operatorname{Re} F(z)$ and $\operatorname{Im} f(z)=\operatorname{Re} G(z)$ for $z$ in $U$. Then $h=(F+i G) / 2$ and $g=(F-i G) / 2$ are analytic in $U$ and $f=h+\bar{g}$. $f$ is locally one-to-one and sense preserving if and only if $\left|g^{\prime}(z)\right|<\left|h^{\prime}(z)\right|$ for $z$ in $U$ [3]. If $h(z)=a_{1} z+a_{2} z^{2}+\ldots, a_{1}>0$, and $g(z)=b_{1} z+b_{2} z^{2}+\ldots$ for $z$ in $U$, it follows that $\left|b_{1}\right|<a_{1}$ and hence $a_{1} f-\bar{b}_{1} \bar{f}$ also belongs to $S_{H}$. Thus, consideration is often restricted to the subclass $S_{H}^{0}$ of $S_{H}$ consisting of those functions in $S_{H}$ with $f_{\bar{z}}(0)=0$.

Since harmonic mappings are not essentially determined by their image domains, various authors have studied subclasses of $S_{H}$, consisting of functions mapping $U$ onto a specific simply connected domain $D$. In particular, Hengartner and Schober [5] considered the case of $D$ being a strip, Abu-Muhanna and Schober [1] considered the case of $D$ being a wedge or a half plane. Cima and the author [2] also considered the case of $D$ being a strip.

In this paper we will study the case of $D$ being the plane $\mathbb{C}$ slit along a ray pointing at the origin. This type of domain is often extremal for certain problems over classes of functions mapping $U$ onto domains that are starlike or convex in one direction. Also, Hengartner and Schober [6] considered the case of $D$ being the plane $\mathbb{C}$ slit along the interval $(-\infty, 0]$. They studied these mappings as they related to minimal surfaces. Our purpose is to study

1991 Mathematics Subject Classification: 30C55, 31A05. 
extreme points and to use the knowledge of extreme points to solve some extremal problems.

Let $a<0$ and $\Omega=\mathbb{C}-(-\infty, a] . S_{H}(U, \Omega)$ is the class of functions $f$ which are univalent sense preserving harmonic maps with $f(U)=\Omega$ and satisfy $f(0)=0, f_{z}(0)>0$ and $f_{\bar{z}}(0)=0$.

In the sequel $F$ and $G$ will be functions analytic in $U$ with $F(0)=$ $G(0)=0$ and $\operatorname{Re} f(z)=\operatorname{Re} F(z)$ and $\operatorname{Re} G(z)=\operatorname{Im} f(z)$ for $z$ in $U$. If $h=(F+i G) / 2$ and $g=(F-i G) / 2$ then $f=h+\bar{g}$ and $\left|g^{\prime}(z)\right|<\left|h^{\prime}(z)\right|$ for $z$ in $U$.

2. The class $S_{H}(U, \Omega)$. Let $\mathcal{P}$ be the class of functions $P(z)$ which are analytic in $U$ with $P(0)=1$ and $\operatorname{Re} P(z)>0$ for $z$ in $U$.

Lemma 1. If $P(z)$ is in $\mathcal{P}$, then

$$
-\frac{1}{2} \leq \operatorname{Re} \int_{0}^{-1} \frac{1+\zeta}{(1-\zeta)^{3}} P(\zeta) d \zeta \leq-\frac{1}{6} .
$$

Proof.

$$
\operatorname{Re} \int_{0}^{-1} \frac{1+\zeta}{(1-\zeta)^{3}} P(\zeta) d \zeta=\int_{0}^{1} \frac{-(1-t)}{(1+t)^{3}} \operatorname{Re} P(-t) d t
$$

However, it is well known that

$$
\frac{1-t}{1+t} \leq \operatorname{Re} P(-t) \leq \frac{1+t}{1-t} .
$$

Thus

$$
\int_{0}^{1} \frac{-1}{(1+t)^{2}} d t \leq \int_{0}^{1} \frac{-(1-t)}{(1+t)^{3}} \operatorname{Re} P(-t) d t \leq \int_{0}^{1} \frac{-(1-t)^{2}}{(1+t)^{4}} d t
$$

and the lemma follows.

We now let $\mathcal{F}$ be the class of functions $f$ which have the form

$$
\begin{aligned}
f(z)=\frac{a}{\operatorname{Re} \int_{0}^{-1} \frac{1+\zeta}{(1-\zeta)^{3}} P(\zeta) d \zeta}\left[\operatorname{Re} \int_{0}^{z} \frac{1+\zeta}{(1-\zeta)^{3}} P(\zeta) d \zeta\right. & \\
& \left.+i \operatorname{Im} \frac{z}{(1-z)^{2}}\right]
\end{aligned}
$$

where $P$ is in $\mathcal{P}$. 
THEOREM 1. If $f \in \mathcal{F}$, then $f$ is harmonic, sense preserving and univalent in $U$ and $f(U)$ is convex in the direction of the real axis with $f(U) \subset \Omega$.

Pro of. Let $f=h+\bar{g}=\operatorname{Re} F+i \operatorname{Re} G$. Then with

$$
A=a / \operatorname{Re} \int_{0}^{-1}\left[(1+\zeta) /(1-\zeta)^{3}\right] P(\zeta) d \zeta
$$

we have

$$
F(z)=A \int_{0}^{z} \frac{1+\zeta}{(1-\zeta)^{3}} P(\zeta) d \zeta \quad \text { and } \quad G(z)=-\frac{i A z}{(1-z)^{2}}
$$

Since

$$
\frac{g^{\prime}(z)}{h^{\prime}(z)}=\frac{F^{\prime}(z)-i G^{\prime}(z)}{F^{\prime}(z)+i G^{\prime}(z)}=\frac{P(z)-1}{P(z)+1},
$$

it follows that $\left|g^{\prime}(z)\right|<\left|h^{\prime}(z)\right|$ for $z$ in $U$. Thus $f$ is locally one-to-one and sense preserving in $U$. Also

$$
h(z)-g(z)=i G(z)=\frac{A z}{(1-z)^{2}}
$$

is convex in the direction of the real axis. By a theorem of Clunie and Sheil-Small [3, Theorem 5.3], $f$ is univalent and $f(U)$ is convex in the direction of the real axis.

Moreover, $f(z)$ is real if and only if $z$ is real. Since $A>0$ and $\operatorname{Re} P(z)>0$ it follows that $f(r)=\operatorname{Re} F(r)$ is increasing on $(-1,1)$ and bounded on $(-1,0)$. Thus $\lim _{r \rightarrow-1^{+}} f(r)$ exists and equals $a$. Moreover, $\lim _{r \rightarrow 1^{-}} f(r)=$ $+\infty$. Thus $f(U)$ omits the interval $(-\infty, a]$. Therefore $f(U) \subset \Omega$.

The next theorem up to translation is contained in [6]. However, for the sake of completeness and since our point of view is somewhat different, we include a proof here.

Theorem 2. $S_{H}(U, \Omega) \subset \mathcal{F}$.

Proof. Let $f$ be in $S_{H}(U, \Omega)$. Since $\Omega$ is convex in the direction of the real axis, by a result of Clunie and Sheil-Small, $h-g=i G$ is univalent and convex in the direction of the real axis.

Let $h(z)=a_{1} z+a_{2} z^{2}+\ldots, a_{1}>0$, and $g(z)=b_{2} z^{2}+\ldots$ Then $G(z)=-i(h(z)-g(z))=-a_{1} i z+\ldots$ Since $f(U)=\Omega, \operatorname{Re} G(z)=\operatorname{Im} f(z)$ is 0 on the boundary of $U$. Since $G$ is convex in the direction of the imaginary axis, it follows that $G(U)$ is $\mathbb{C}$ slit along one or two infinite rays along the imaginary axis. Thus $G(z) /\left(-a_{1} i\right)$ maps $U$ onto $\mathbb{C}$ slit along one or two infinite rays along the real axis. However, $G(z) /\left(-a_{1} i\right)$ is a member of the class $S$ of functions $f$ analytic and univalent in $U$ and normalized by $f(0)=f^{\prime}(0)-1=0$. Making use of subordination arguments, it follows 
that $G(z) /\left(-a_{1} i\right)$ has the form

$$
\frac{G(z)}{-a_{1} i}=\frac{z}{1+c z+z^{2}}, \quad-2 \leq c \leq 2,
$$

and hence $\operatorname{Im} f(r)=\operatorname{Re} G(r)=0$ for $-1<r<1$. Since $f_{z}(0)>0$, the function $f$ is increasing on $(-1,1)$, so that $\lim _{r \rightarrow-1^{+}} f(r)=a$ and $\lim _{r \rightarrow 1^{-}} f(r)=+\infty$.

Now if $f=h+\bar{g}$, then $h^{\prime}-g^{\prime}=i G^{\prime}$ and

$$
\frac{h^{\prime}+g^{\prime}}{h^{\prime}-g^{\prime}}=\frac{1+g^{\prime} / h^{\prime}}{1-g^{\prime} / h^{\prime}} .
$$

Since $\left|g^{\prime}(z) / h^{\prime}(z)\right|<1$ for $z$ in $U$, it follows that

$$
\frac{h^{\prime}+g^{\prime}}{h^{\prime}-g^{\prime}}=P,
$$

where $P$ is in $\mathcal{P}$. Thus $h^{\prime}+g^{\prime}=\left(h^{\prime}-g^{\prime}\right) P=i G^{\prime} P$, and

$$
F(z)=h(z)+g(z)=\int_{0}^{z} i G^{\prime}(\zeta) P(\zeta) d \zeta
$$

Now suppose $G(z)=-a_{1} i z /\left(1+c z+z^{2}\right),-2<c \leq 2$. Then

$$
F(z)=a_{1} \int_{0}^{z} \frac{1-\zeta^{2}}{\left(1+c \zeta+\zeta^{2}\right)^{2}} P(\zeta) d \zeta .
$$

If $0<r<1$, then

$$
\begin{aligned}
f(r)=\operatorname{Re} f(r)=\operatorname{Re} F(r) & =a_{1} \int_{0}^{r} \frac{1-t^{2}}{\left(1+c t+t^{2}\right)^{2}} \operatorname{Re} P(t) d t \\
& \leq a_{1} \int_{0}^{r} \frac{\left(1-t^{2}\right)(1+t)}{\left(1+c t+t^{2}\right)^{2}(1-t)} d t \\
& =a_{1} \int_{0}^{r} \frac{(1+t)^{2}}{\left(1+c t+t^{2}\right)^{2}} d t \leq M
\end{aligned}
$$

for some $M$, since $-2<c \leq 2$. However, this is impossible since $\lim _{r \rightarrow 1^{-}} f(r)=+\infty$.

Thus the only possibility is that $G(z)=-a_{1} i z /(1-z)^{2}$, and

$$
F(z)=a_{1} \int_{0}^{z} \frac{1+\zeta}{(1-\zeta)^{3}} P(\zeta) d \zeta .
$$


Thus,

$$
a=\lim _{r \rightarrow-1^{+}} f(r)=a_{1} \operatorname{Re} \int_{0}^{-1} \frac{1+\zeta}{(1-\zeta)^{3}} P(\zeta) d \zeta,
$$

and the theorem follows.

Theorem 3. $\overline{S_{H}(U, \Omega)}=\mathcal{F}$.

Proof. Let $f(z)$ have the form (1.1), and let $r_{n}$ be a sequence with $0<r_{n}<1$ and $\lim r_{n}=1$. Let $P_{n}(z)=P\left(r_{n} z\right)$, and denote by $f_{n}(z)$ the function obtained from (1.1) by replacing $P(z)$ by $P_{n}(z)$. We claim that $f_{n}$ is in $S_{H}(U, \Omega)$. To see this, let

$$
\begin{aligned}
A & =a / \operatorname{Re} \int_{0}^{-1}\left[(1+\zeta) /(1-\zeta)^{3}\right] P_{n}(\zeta) d \zeta, \\
F_{n}(z) & =A \int_{0}^{z}\left[(1+\zeta) /(1-\zeta)^{3}\right] P_{n}(\zeta) d \zeta .
\end{aligned}
$$

There exists $\delta>0$ so that we may write for $|z-1|<\delta$,

$$
P_{n}(z)=P_{n}(1)+P_{n}^{\prime}(1)(z-1)+\frac{P_{n}^{\prime \prime}(1)}{2}(z-1)^{2}+\ldots
$$

Then, for $|z-1|<\delta$,

$$
\begin{aligned}
F_{n}^{\prime}(z) & =A \frac{1+z}{(1-z)^{3}} P_{n}(z) \\
& =A\left[\frac{-2 P_{n}(1)}{(z-1)^{3}}-\frac{2 P_{n}^{\prime}(1)+P_{n}(1)}{(z-1)^{2}}-\frac{P_{n}^{\prime \prime}(1)+P_{n}^{\prime}(1)}{z-1}+\ldots\right] .
\end{aligned}
$$

Let $D=\{z:|z-1|<\delta\}-\{z: 1 \leq z \leq 1+\delta\}$. If $1-\delta<c<1$, then for $z$ in $D$

$$
F_{n}(z)-F_{n}(c)=\int_{c}^{z} F_{n}^{\prime}(\zeta) d \zeta
$$

where the path of integration is in $D$. This gives, for $z$ in $D$,

$$
\begin{aligned}
F_{n}(z)=A\left[\frac{2 P_{n}(1)}{(z-1)^{2}}+\right. & \frac{2 P_{n}^{\prime}(1)+P_{n}(1)}{z-1} \\
& \left.-\left(P_{n}^{\prime \prime}(1)+P_{n}^{\prime}(1)\right) \log (z-1)+\sum_{j=0}^{\infty} c_{j}(z-1)^{j}\right]
\end{aligned}
$$

where $\sum_{j=0}^{\infty} c_{j}(z-1)^{j}$ converges for $|z-1|<\delta$ and $\log (z-1)=\ln |z-1|+$ $i \arg (z-1), 0<\arg (z-1)<2 \pi$. That is, for $z$ in $D, F_{n}$ has the form

$$
F_{n}(z)=A\left[\frac{c}{(z-1)^{2}}+\frac{d}{(z-1)}+e \log (z-1)+q(z)\right],
$$


where $\operatorname{Re} c=2 \operatorname{Re} P_{n}(1)>0$ and $q(z)$ is analytic at $z=1$. Thus, for $z$ in $D$,

$$
\begin{aligned}
\operatorname{Re} f_{n}(z) & =\operatorname{Re} F_{n}(z) \\
=A\left[\operatorname{Re}\left(\frac{c}{(z-1)^{2}}+\frac{d}{(z-1)}\right)+\operatorname{Re}(e) \ln |z-1|\right. & \quad-\operatorname{Im}(e) \arg (z-1)+\operatorname{Re} q(z)] .
\end{aligned}
$$

We want to prove that $f_{n}$ cannot have a nonreal finite cluster point at $z=1$. To see this, suppose $z_{j}=1+t_{j} e^{i \theta_{j}}$ is in $U$ with $t_{j}>0$ and $\lim t_{j}=0$ and is such that

$$
\lim _{j \rightarrow \infty} \operatorname{Im}\left[\frac{z_{j}}{\left(1-z_{j}\right)^{2}}\right]=l,
$$

where $l$ is finite and $l \neq 0$. Then

$$
\lim _{j \rightarrow \infty} \frac{-\left(\sin 2 \theta_{j}+t_{j} \sin \theta_{j}\right)}{t_{j}^{2}}=l \neq 0 .
$$

This implies that $\left(\sin 2 \theta_{j}+t_{j} \sin \theta_{j}\right)$ approaches 0 , which in turn implies that $\sin 2 \theta_{j}$ approaches 0 . Thus $e^{-2 i \theta_{j}}$ approaches \pm 1 . Therefore, $\operatorname{Re}\left(c e^{-i 2 \theta_{j}}\right)$ approaches $\pm \operatorname{Re} c \neq 0$. It now follows that

$$
\begin{array}{r}
\left|\operatorname{Re} f_{n}\left(z_{j}\right)\right|=\mid \frac{\operatorname{Re}\left(c e^{-i 2 \theta_{j}}\right)+t_{j} \operatorname{Re}\left(d e^{-i \theta_{j}}\right)+\operatorname{Re}(e) t_{j}^{2} \ln \left(t_{j}\right)}{t_{j}^{2}} \\
-(\operatorname{Im} e) \arg \left(z_{j}-1\right)+\operatorname{Re} q\left(z_{j}\right) \mid
\end{array}
$$

approaches $+\infty$ as $n$ approaches $+\infty$. Thus $f_{n}$ has no finite nonreal cluster points at $z=1$. At all other points of $|z|=1$, the finite cluster points of $f_{n}$ are real. Since $f_{n}(U) \subset \Omega$ and $\lim _{r \rightarrow-1} f_{n}(r)=a$, it follows that $f_{n}(U)=\Omega$.

Thus $f_{n}$ is in $S_{H}(U, \Omega)$ and hence $f$ is in $\overline{S_{H}(U, \Omega)}$. Since $\mathcal{F}$ is closed under uniform limits on compact subsets of $U$, it follows that $\mathcal{F}=\overline{S_{H}(U, \Omega)}$.

3. Extreme points of $\mathcal{F}$. If $P \in \mathcal{P}$, then it is known [4] that

$$
P(z)=\int_{|\eta|=1} \frac{1+\eta z}{1-\eta z} d \mu(\eta)
$$

where $\mu$ is a probability measure on $X=\{\eta:|\eta|=1\}$. Thus if $f$ is in $\mathcal{F}$, 
there is a probability measure $\mu$ on $X$ so that

$$
\begin{aligned}
& f(z)=\frac{a}{\operatorname{Re} \int_{|\eta|=1} k(-1, \eta) d \mu(\eta)} \\
& \times\left[\operatorname{Re} \int_{|\eta|=1} k(z, \eta) d \mu(\eta)+i \operatorname{Im} \frac{z}{(1-z)^{2}}\right]
\end{aligned}
$$

where

$$
\begin{aligned}
k(z, \eta)= & \int_{0}^{z} \frac{(1+\zeta)(1+\eta \zeta)}{(1-\zeta)^{3}(1-\eta \zeta)} d \zeta \\
& = \begin{cases}\frac{2 \eta(1+\eta)}{(1-\eta)^{3}} \log \left(\frac{1-\eta z}{1-z}\right)-\frac{\left(1+4 \eta-\eta^{2}\right) z}{(1-\eta)^{2}(1-z)} \\
+\frac{(1+\eta)\left(2 z-z^{2}\right)}{(1-\eta)(1-z)^{2}}, \quad \eta \neq 1, \\
\frac{z+\frac{1}{3} z^{3}}{(1-z)^{3}}, \quad \eta=1 .\end{cases}
\end{aligned}
$$

The extreme points of $\mathcal{F}$ are easily obtained by making use of a property of a nonlinear homeomorphism observed by Szapiel [7].

Lemma 2 [7]. Suppose $X$ is a convex linear Hausdorff space, $\phi: X \rightarrow \mathbb{C}$ is homogeneous, $c \in \mathbb{C} \backslash\{0\}$ and $A$ is a compact convex subset of $\phi^{-1}(c)$. Let $\psi: A \rightarrow \mathbb{R}$ be affine continuous with $0 \notin \psi(A)$ and let $B=\{a / \psi(a): a \in A\}$. Then

1) $B$ is compact convex,

2) the map $a \rightarrow a / \psi(a)$ is a homeomorphism of $A$ onto $B$,

3) $E B=\{a / \psi(a): a \in E A\}$, where $E D$ means the set of all extreme points of $D$.

Proof. For the sake of completeness we include the proof as communicated to me by the referee. We observe that if $a_{1}$ and $a_{2}$ are in $A$ and $a_{1} / \psi\left(a_{1}\right)=a_{2} / \psi\left(a_{2}\right)$, then $c / \psi\left(a_{1}\right)=\phi\left(a_{1} / \psi\left(a_{1}\right)\right)=\phi\left(a_{2} / \psi\left(a_{2}\right)\right)=$ $c / \psi\left(a_{2}\right)$. Thus $\psi\left(a_{1}\right)=\psi\left(a_{2}\right)$ and hence $a_{1}=a_{2}$. Next we note that $\left(\lambda_{1} a_{1}+\lambda_{2} a_{2}\right) / \psi\left(\lambda_{1} a_{1}+\lambda_{2} a_{2}\right)=\mu_{1} a_{1} / \psi\left(a_{1}\right)+\mu_{2} a_{2} / \psi\left(a_{2}\right)$, where

$$
\mu_{j}=\lambda_{j} \psi\left(a_{j}\right) / \psi\left(\lambda_{1} a_{1}+\lambda_{2} a_{2}\right), \quad j=1,2 .
$$

Thus if $\lambda_{j}>0$ with $j=1,2$, and $\lambda_{1}+\lambda_{2}=1$ then $\mu_{j}>0$ with $\mu_{1}+\mu_{2}=1$. If $\mu_{j}>0, j=1,2$, with $\mu_{1}+\mu_{2}=1$, we seek $\lambda_{j}>0, j=1,2$, with $\lambda_{1}+\lambda_{2}=1$ such that equation (3.4) is satisfied. It is easily verified that $\lambda_{1}=\mu_{1} \psi\left(a_{2}\right) /\left[\mu_{1} \psi\left(a_{2}\right)+\mu_{2} \psi\left(a_{1}\right)\right]$ and $\lambda_{2}=\mu_{2} \psi\left(a_{1}\right) /\left[\mu_{1} \psi\left(a_{2}\right)+\mu_{2} \psi\left(a_{1}\right)\right]$ satisfy the requirements. Thus $B$ is convex. 
Next suppose $a \notin E A$. Then $a=\lambda_{1} a_{1}+\lambda_{2} a_{2}$ with $\lambda_{j}>0, j=1,2$, $\lambda_{1}+\lambda_{2}=1$ and $a_{1} \neq a_{2}$. Thus

$$
\frac{a}{\psi(a)}=\frac{\lambda_{1} a_{1}+\lambda_{2} a_{2}}{\psi\left(\lambda_{1} a_{1}+\lambda_{2} a_{2}\right)}=\mu_{1} \frac{a_{1}}{\psi\left(a_{1}\right)}+\mu_{2} \frac{a_{2}}{\psi\left(a_{2}\right)} .
$$

So $a / \psi(a) \notin E B$.

Conversely, suppose $a / \psi(a) \notin E B$. Then there exist $a_{1} \neq a_{2}$ in $A$ and $\mu_{j}>0, j=1,2$, with $\mu_{1}+\mu_{2}=1$ so that

$$
\frac{a}{\psi(a)}=\mu_{1} \frac{a_{1}}{\psi\left(a_{1}\right)}+\mu_{2} \frac{a_{2}}{\psi\left(a_{2}\right)}=\frac{\lambda_{1} a_{1}+\lambda_{2} a_{2}}{\psi\left(\lambda_{1} a_{1}+\lambda_{2} a_{2}\right)} .
$$

Since the map $a \rightarrow a / \psi(a)$ is a homeomorphism, it follows that $a=\lambda_{1} a_{1}+$ $\lambda_{2} a_{2}$ and hence $a \notin E A$. Therefore $E B=\{a / \psi(a): a \in E A\}$.

We apply Lemma 2 with

$$
\begin{gathered}
Q_{P}(z)=\operatorname{Re}\left[\int_{0}^{z}(1+\zeta)(1-\zeta)^{-3} P(\zeta) d \zeta\right]+i \operatorname{Im}\left[z(1-z)^{-2}\right] \\
A=\left\{Q_{P}: P \in \mathcal{P}\right), \phi(f)=f_{z}(0), c=1, \psi\left(Q_{P}\right)=Q_{P}(-1) / a
\end{gathered}
$$

Then $\mathcal{F}=B$ is convex and $E B=\{f(z) / f(-1): f \in E A\}$. However, the map $Q_{P} \rightarrow P$ is a linear homeomorphism between $A$ and $\mathcal{P}$. Since $E \mathcal{P}=\{(1+\mu z) /(1-\mu z):|\mu|=1\}[4]$, we obtain the following theorem.

TheOrem 4. The extreme points of $\mathcal{F}$ are

$$
f_{\eta}(z)=\frac{a}{\operatorname{Re} k(-1, \eta)}\left[\operatorname{Re} k(z, \eta)+i \operatorname{Im} \frac{z}{(1-z)^{2}}\right], \quad|\eta|=1 .
$$

4. The mapping properties of extreme points. If $\eta=e^{i \beta}$, then

$$
\begin{aligned}
\operatorname{Re} k(z, \eta)= & \frac{\cot (\beta / 2)}{2 \sin ^{2}(\beta / 2)} \arg \left(\frac{1-e^{i \beta} z}{1-z}\right) \\
& -\cot (\beta / 2) \operatorname{Im} \frac{z}{(1-z)^{2}}+\frac{1}{\sin ^{2}(\beta / 2)} \operatorname{Re} \frac{z}{1-z}
\end{aligned}
$$

and

$$
f_{\eta}(z)=\frac{a}{\frac{(\beta / 2) \cot (\beta / 2)}{2 \sin ^{2}(\beta / 2)}-\frac{1}{2 \sin ^{2}(\beta / 2)}}\left[\operatorname{Re} k(z, \eta)+i \operatorname{Im} \frac{z}{(1-z)^{2}}\right] .
$$

Suppose $\eta=e^{i \beta}, 0<\beta<\pi$. If $\zeta$ is on the open arc of the unit circle going from 1 to $\eta$ to -1 to $\bar{\eta}$ in the counterclockwise direction, then $\arg (1-\eta \zeta) /(1-\zeta)=\beta / 2$. For these $\zeta$,

$$
\lim _{z \rightarrow \zeta} f_{\eta}(z)=a .
$$


If $\zeta$ is on the open arc from $\bar{\eta}$ to 1 , then $\arg (1-\eta \zeta) /(1-\zeta)=\beta / 2-\pi$ and we obtain

$$
\lim _{z \rightarrow \zeta} f_{\eta}(z)=a-\frac{\pi a \cot (\beta / 2)}{(\beta / 2) \cot (\beta / 2)-1}=b<a .
$$

The cluster set of $\arg (1-\eta z) /(1-z)$ at $\bar{\eta}$ is the interval $[\beta / 2-\pi, \beta / 2]$. Thus, the cluster set of $f_{\eta}(z)$ at $\bar{\eta}$ is the interval $[b, a]$.

We now use a technique similar to that used in Example 5.4 of [3]. Let $(1+z) /(1-z)=u+i v$ and note

$$
\begin{aligned}
z /(1-z) & =\frac{1}{2}[(1+z) /(1-z)-1], \\
((1+z) /(1-z))^{2} & =4 z /(1-z)^{2}+1, \\
(1-\eta z) /(1-z) & =[(1-\eta) / 2](1+z) /(1-z)+(1+\eta) / 2, \\
\operatorname{Im}\left[z /(1-z)^{2}\right] & =\frac{1}{4} \operatorname{Im}(u+i v)^{2} .
\end{aligned}
$$

Using these observations, we obtain

$$
\begin{aligned}
f_{\eta}(z)= & \frac{a}{\operatorname{Re} k(-1, \eta)} \\
& \times\left[\frac{\cot (\beta / 2)}{2 \sin ^{2}(\beta / 2)} \arg \left[\left(1+e^{i \beta}\right)(1+v \tan (\beta / 2)-i u \tan (\beta / 2))\right]\right. \\
& \left.-\frac{u v}{2} \cot (\beta / 2)+\frac{u-1}{2 \sin ^{2}(\beta / 2)}+i \frac{u v}{2}\right]=x+i y .
\end{aligned}
$$

If $u v=2 c, u>0$, then $y=a c / \operatorname{Re} k(-1, \eta)$ and

$$
\begin{aligned}
x= & \frac{a}{\operatorname{Re} k(-1, \eta)} \\
& \times\left[\frac{\cot (\beta / 2)}{2 \sin ^{2}(\beta / 2)} \arg \left[\left(1+e^{i \beta}\right)\left(1+\frac{2 c \tan (\beta / 2)}{u}-i u \tan (\beta / 2)\right)\right]\right. \\
& \left.-c \cot (\beta / 2)+\frac{u-1}{2 \sin ^{2}(\beta / 2)}\right] .
\end{aligned}
$$

Now suppose that $c>0$. Then $\lim _{u \rightarrow+\infty} x=+\infty$, and

$$
\begin{aligned}
\lim _{u \rightarrow 0} x= & \frac{a}{\operatorname{Re} k(-1, \eta)} \\
& \times\left[\frac{\cot (\beta / 2)}{2 \sin ^{2}(\beta / 2)} \arg \left(1+e^{i \beta}\right)-c \cot (\beta / 2)-\frac{1}{2 \sin ^{2}(\beta / 2)}\right] \\
= & a-\frac{c a \cot (\beta / 2)}{\operatorname{Re} k(-1, \eta)} .
\end{aligned}
$$

A calculation gives $d x / d u>0$ for any $c$. Thus $x$ is increasing. Thus if $c>0, x$ takes on all values in the interval $(a-c a \cot (\beta / 2) / \operatorname{Re} k(-1, \eta),+\infty)$. 
It follows that $f_{\eta}(z)$ covers the horizontal line segment $y=a c / \operatorname{Re} k(-1, \eta)$, with left end point on the line $y=-[\tan (\beta / 2)](x-a)$.

By the same reasoning, if $c<0, f_{\eta}(z)$ covers the horizontal line segment $y=a c / \operatorname{Re} k(-1, \eta)$ with left end point on the line $y=-[\tan (\beta / 2)](x-b)$.

If $c=0, u v=0$. Since $u>0$, it follows that $v=0$ and

$$
\begin{aligned}
x= & \frac{a}{\operatorname{Re} k(-1, \eta)} \\
& \times\left[\frac{\cot (\beta / 2)}{2 \sin ^{2}(\beta / 2)} \arg \left[\left(1+e^{i \beta}\right)(1-i u \tan (\beta / 2))\right]+\frac{u-1}{2 \sin ^{2}(\beta / 2)}\right] .
\end{aligned}
$$

Thus,

$$
\lim _{u \rightarrow 0} x=a \text { and } \quad \lim _{u \rightarrow+\infty} x=+\infty .
$$

Since $x$ is increasing, $f_{\eta}$ covers the interval $(a,+\infty)$ on the real axis and no other part of the real axis. It follows that $f_{\eta}(z)$ maps $U$ onto the domain given by the shaded region in Figure 4.1.

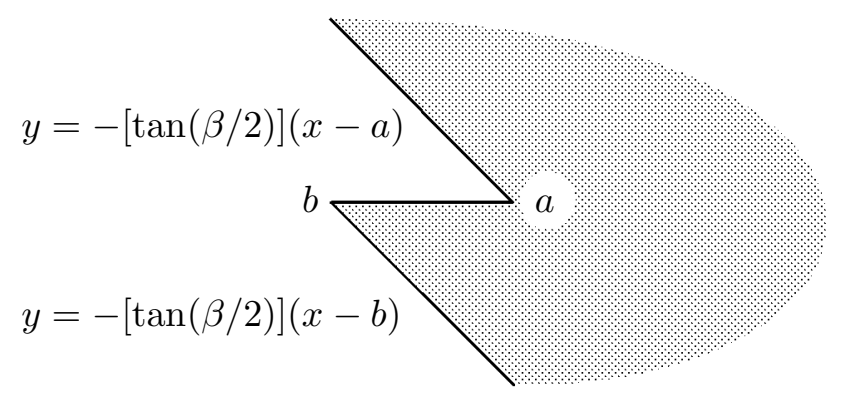

Fig. 4.1

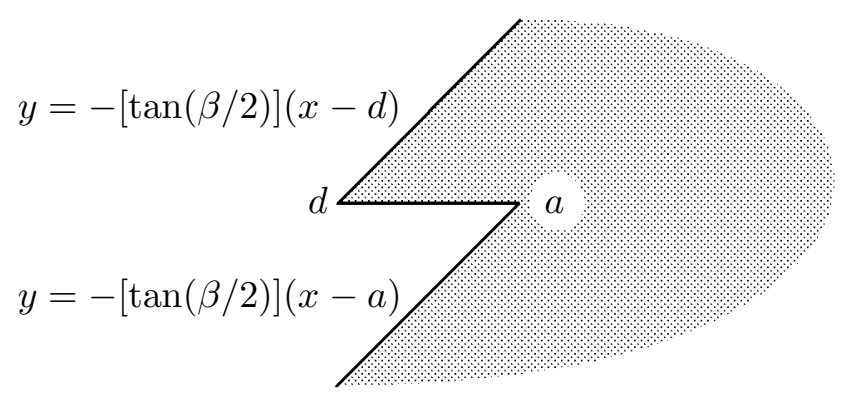

Fig. 4.2

Similarly if $-\pi<\beta<0, f_{\eta}(z)$ maps $U$ onto the domain pictured in Figure 4.2, where

$$
d=a+\frac{a \pi \cot (\beta / 2)}{(\beta / 2) \cot (\beta / 2)-1}<a .
$$


5. Applications. In this section we will use our knowledge of extreme points to solve some extremal problems on $\overline{S_{H}(U, \Omega)}$.

LEMMA 3. If $|\eta|=1$, then

$$
|\operatorname{Re} k(-1, \eta)| \geq|\operatorname{Re} k(-1,1)|=1 / 6 .
$$

Proof.

$$
k(-1, \eta)= \begin{cases}\frac{2 \eta(1+\eta)}{(1-\eta)^{3}} \log \left(\frac{1+\eta}{2}\right)+\frac{1+4 \eta-\eta^{2}}{2(1-\eta)^{2}}-\frac{3}{4} \cdot \frac{1+\eta}{1-\eta}, & \eta \neq 1, \\ -1 / 6, & \eta=1 .\end{cases}
$$

If $\eta=e^{i \theta}$, then

$$
\begin{aligned}
k\left(-1, e^{i \theta}\right)= & \frac{2 e^{i \theta}}{\left(1-e^{i \theta}\right)^{2}} \cdot \frac{1+e^{i \theta}}{1-e^{i \theta}} \log \left(\frac{1+e^{i \theta}}{2}\right) \\
& +\frac{1+4 e^{i \theta}-e^{i 2 \theta}}{2\left(1-e^{i \theta}\right)^{2}}-\frac{3}{4} \cdot \frac{1+e^{i \theta}}{1-e^{i \theta}} \\
= & \frac{2}{\left(e^{-i \theta / 2}-e^{i \theta / 2}\right)^{2}} \cdot \frac{e^{-i \theta / 2}+e^{i \theta / 2}}{e^{-i \theta / 2}-e^{i \theta / 2}} \log \left(\frac{1+e^{i \theta}}{2}\right) \\
& +\frac{e^{-i \theta}+4-e^{i \theta}}{2\left(e^{-i \theta / 2}-e^{i \theta / 2}\right)^{2}}-\frac{3}{4} \cdot \frac{e^{-i \theta / 2}+e^{i \theta / 2}}{e^{-i \theta / 2}-e^{i \theta / 2}} \\
= & \frac{-i \cos (\theta / 2)}{2 \sin ^{3}(\theta / 2)} \log \left(\frac{1+e^{i \theta}}{2}\right)-\frac{2-i \sin \theta}{4 \sin ^{2}(\theta / 2)}-\frac{3 i \cos (\theta / 2)}{4 \sin (\theta / 2)} .
\end{aligned}
$$

Thus

$$
\begin{aligned}
\operatorname{Re} k\left(-1, e^{i \theta}\right) & =\frac{\cos (\theta / 2)}{2 \sin ^{3}(\theta / 2)} \arg \left(\frac{1+e^{i \theta}}{2}\right)-\frac{1}{2 \sin ^{2}(\theta / 2)} \\
& =\frac{(\theta / 2) \cos (\theta / 2)}{2 \sin ^{3}(\theta / 2)}-\frac{1}{2 \sin ^{2}(\theta / 2)} \\
& =\frac{(\theta / 2) \cos (\theta / 2)-\sin (\theta / 2)}{2 \sin ^{3}(\theta / 2)} .
\end{aligned}
$$

Let $q(\theta)=[(\theta / 2) \cos (\theta / 2)-\sin (\theta / 2)] /\left[2 \sin ^{3}(\theta / 2)\right]$ if $\theta \neq 0$ and $q(0)=$ $-1 / 6$. We want to find the maximum of $q(\theta)$ in $[-\pi, \pi]$, or equivalently the maximum of $h(\phi)$ in $[-\pi / 2, \pi / 2]$, where $h(\phi)=(\phi \cos \phi-\sin \phi) /\left(2 \sin ^{3} \phi\right)$ for $\phi \neq 0$ and $h(0)=-1 / 6$. Since $h(-\phi)=h(\phi)$, we need to consider $h(\phi)$ in $[0, \pi / 2]$. Let $g(\phi)=6 \phi \cos \phi-6 \sin \phi+2 \sin ^{3} \phi$ for $0 \leq \phi \leq \pi / 2$. Then

$$
\begin{aligned}
g^{\prime}(\phi) & =-6 \phi \sin \phi+6 \sin ^{2} \phi \cos \phi \\
& =3 \sin \phi(\sin 2 \phi-2 \phi) \leq 0 .
\end{aligned}
$$

Thus $g(\phi) \leq g(0)=0$. It follows that $h(\phi) \leq-1 / 6$ in $[0, \pi / 2]$ and thus 
$q(\theta) \leq-1 / 6$ on $[-\pi, \pi]$. Therefore

$$
|\operatorname{Re} k(-1, \eta)|=|q(\theta)| \geq 1 / 6=|\operatorname{Re} k(-1,1)| .
$$

TheOREM 5. Let $f(z)=h(z)+\overline{g(z)}$ be in $\overline{S_{H}(U, \Omega)}$. If $h(z)=\sum_{n=1}^{\infty} a_{n} z^{n}$ and $g(z)=\sum_{n=2}^{\infty} b_{n} z^{n}$, then

$$
\begin{aligned}
\left|a_{n}\right| & \leq(n+1)(2 n+1)|a|, \\
\left|b_{n}\right| & \leq(n-1)(2 n-1)|a|, \\
|| a_{n}|-| b_{n}|| & \leq\left|a_{n}-b_{n}\right| \leq 6|a| n .
\end{aligned}
$$

Equality is attained in (5.1), (5.2) and (5.3) for all $n$ by

$$
f(z)=-6 a\left[\operatorname{Re} \frac{z+\frac{1}{3} z^{3}}{(1-z)^{3}}+i \operatorname{Im} \frac{z}{(1-z)^{2}}\right] .
$$

Proof. We need only prove (5.1)-(5.3) for the extreme points of $\overline{S_{H}(U, \Omega)}$. Let $f_{\eta}(z)=A\left[\operatorname{Re} k(z, \eta)+i \operatorname{Im} z /(1-z)^{2}\right], A=a / \operatorname{Re} k(-1, \eta)$. In our notation $F(z)=A k(z, \eta)$ and $G(z)=-A i z /(1-z)^{2}$. Thus

$$
\begin{aligned}
& h(z)=\frac{F(z)+i G(z)}{2}=\frac{A}{2}\left[k(z, \eta)+\frac{z}{(1-z)^{2}}\right]=\sum_{n=1}^{\infty} a_{n} z^{n}, \\
& g(z)=\frac{F(z)-i G(z)}{2}=\frac{A}{2}\left[k(z, \eta)-\frac{z}{(1-z)^{2}}\right]=\sum_{n=2}^{\infty} b_{n} z^{n} .
\end{aligned}
$$

Thus if $\eta \neq 1$, then

$$
\begin{aligned}
h(z)=\frac{A}{2}\left[\sum_{n=1}^{\infty} \frac{2 \eta(1+\eta)\left(1-\eta^{n}\right)}{(1-\eta)^{3} n}\right. & z^{n}-\sum_{n=1}^{\infty} \frac{1+4 \eta-\eta^{2}}{(1-\eta)^{2}} z^{n} \\
& \left.+\sum_{n=1}^{\infty} \frac{(n+1)(1+\eta)}{1-\eta} z^{n}+\sum_{n=1}^{\infty} n z^{n}\right] .
\end{aligned}
$$

Therefore

$$
\begin{array}{r}
a_{n}=\frac{A}{2}\left[\frac{2 \eta(1+\eta)\left(1-\eta^{n}\right)}{n(1-\eta)^{3}}-\frac{1+4 \eta-\eta^{2}}{(1-\eta)^{2}}+\frac{(n+1)(1+\eta)}{1-\eta}+n\right] \\
=\frac{A}{2}\left[\frac{2 \eta(1+\eta)\left(1+\eta+\eta^{2}+\ldots+\eta^{n-1}\right)}{n(1-\eta)^{2}}-\frac{1+4 \eta-\eta^{2}}{(1-\eta)^{2}}\right. \\
\left.+\frac{(n+1)(1+\eta)}{1-\eta}+n\right] \\
=\frac{A}{2}\left[\frac{n^{2}+(2-4 n) \eta+\left(4-n^{2}\right) \eta^{2}+4 \eta^{3}+\ldots+4 \eta^{n}+2 \eta^{n+1}}{n(1-\eta)^{2}}+n\right]
\end{array}
$$




$$
\begin{aligned}
& =\frac{A}{2}\left[\frac{(1-\eta)^{2}\left(n^{2}+\sum_{k=1}^{n-1} 2(n-k)^{2} \eta^{k}\right)}{n(1-\eta)^{2}}+n\right] \\
& =A\left[\frac{n^{2}+\sum_{k=1}^{n-1}(n-k)^{2} \eta^{k}}{n}\right] .
\end{aligned}
$$

Similarly, for $n \geq 2$

$$
b_{n}=\frac{A}{2}\left[\frac{n^{2}+\sum_{k=1}^{n-1} 2(n-k)^{2} \eta^{k}}{n}-n\right]=A\left[\frac{\sum_{k=1}^{n-1}(n-k)^{2} \eta^{k}}{n}\right] .
$$

We note that (5.4) and (5.5) also hold for $\eta=1$.

Now by Lemma 3

$$
|A|=\frac{|a|}{|\operatorname{Re} k(-1, \eta)|} \leq \frac{|a|}{|\operatorname{Re} k(-1,1)|}=6|a| .
$$

Thus from (5.4) and (5.5) we obtain

$$
\begin{aligned}
\left|a_{n}\right| & \leq 6|a| \frac{n^{2}+\sum_{k=1}^{n-1}(n-k)^{2}}{n}=\frac{6|a|}{n} \cdot \frac{n(n+1)(2 n+1)}{6} \\
& =(n+1)(2 n+1)|a|
\end{aligned}
$$

with equality when $\eta=1$, and

$$
\begin{aligned}
\left|b_{n}\right| & \leq \frac{6|a|}{n} \sum_{k=1}^{n-1}(n-k)^{2}=\frac{6|a|}{n} \cdot \frac{(n-1) n(2 n-1)}{6} \\
& =(n-1)(2 n-1)|a|
\end{aligned}
$$

with equality for $\eta=1$.

To obtain (5.3) we note that for an extreme point $f_{\eta}(z)$ we have $a_{n}-b_{n}=$ An.

Theorem 6. If $f=h+\bar{g}$ is in $S_{H}(U, \Omega)$, then

$$
\left|f_{z}(z)\right|=\left|h^{\prime}(z)\right| \leq \frac{6|a|}{1-|z|}\left|\frac{1+z}{(1-z)^{3}}\right| \leq \frac{6(1+|z|)|a|}{(1-|z|)^{4}} .
$$

Equality occurs for $z$ real and positive and

$$
f(z)=-6 a\left[\operatorname{Re} \frac{z+\frac{1}{3} z^{3}}{(1-z)^{3}}+i \operatorname{Im} \frac{z}{(1-z)^{2}}\right] .
$$

Proof. We need only consider extreme points $f_{\eta}(z)$. In this case

$$
\begin{aligned}
h(z) & =\frac{A}{2}\left[k(z, \eta)+\frac{z}{(1-z)^{2}}\right] \\
& =\frac{A}{2}\left[\frac{2 \eta(1+\eta)}{(1-\eta)^{3}} \log \left(\frac{1-\eta z}{1-z}\right)-\frac{4 \eta}{(1-\eta)^{2}} \cdot \frac{z}{1-z}+\frac{2}{1-\eta} \cdot \frac{z}{(1-z)^{2}}\right] .
\end{aligned}
$$


After straightforward computations we get

$$
\begin{aligned}
h^{\prime}(z) & =\frac{a}{\operatorname{Re} k(-1, \eta)} \cdot \frac{(1+z)}{(1-z)^{3}(1-\eta z)}, \\
\left|h^{\prime}(z)\right| & =\frac{|a|}{|\operatorname{Re} k(-1, \eta)||1-\eta z|}\left|\frac{1+z}{(1-z)^{3}}\right| \\
& \leq \frac{6|a|}{1-|z|}\left|\frac{1+z}{(1-z)^{3}}\right| \leq \frac{6|a|(1+|z|)}{(1-|z|)^{4}} .
\end{aligned}
$$

Acknowledgement. The author would like to thank the referee for a careful reading of the manuscript and for bringing reference [7] to his attention.

\section{References}

[1] Y. Abu-Muhanna and G. Schober, Harmonic mappings onto convex domains, Canad. J. Math. 39 (1987), 1489-1530.

[2] J. A. Cima and A. E. Livingston, Integral smoothness properties of some harmonic mappings, Complex Variables 11 (1989), 95-110.

[3] J. Clunie and T. Sheil-Small, Harmonic univalent functions, Ann. Acad. Sci. Fenn. Ser. AI 9 (1984), 3-25.

[4] D. J. Hallenbeck and T. H. MacGregor, Linear Problems and Convexity Techniques in Geometric Function Theory, Monographs and Studies in Math. 22, Pitman, 1984.

[5] W. Hengartner and G. Schober, Univalent harmonic functions, Trans. Amer. Math. Soc. 299 (1987), 1-31.

[6] -, - Curvature estimates for some minimal surfaces, in: Complex Analysis, Birkhäuser, 1988, 87-100.

[7] W. Szapiel, Extremal problems for convex sets. Applications to holomorphic functions, Dissertation XXXVII, UMCS Press, Lublin 1986 (in Polish).

DEPARTMENT OF MATHEMATICAL SCIENCES

UNIVERSITY OF DELAWARE

501 EWING HALL

NEWARK, DELAWARE 19716

U.S.A.

Reçu par la Rédaction le 14.1.1991

Révisé le 5.7.1991 